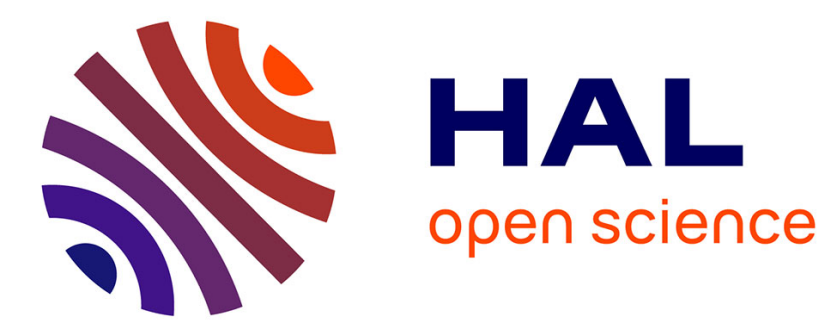

\title{
Impact of Robot Initiative on Human-Robot Collaboration
}

\author{
Thibaut Munzer, Yoan Mollard, Manuel Lopes
}

\section{To cite this version:}

Thibaut Munzer, Yoan Mollard, Manuel Lopes. Impact of Robot Initiative on Human-Robot Collaboration. HRI 2017 - ACM/IEEE International Conference on Human-Robot Interaction, Mar 2017, Vienne, Austria. pp. 217-218, 10.1145/3029798.3038373 . hal-01644010

\section{HAL Id: hal-01644010 https://hal.science/hal-01644010}

Submitted on 21 Nov 2017

HAL is a multi-disciplinary open access archive for the deposit and dissemination of scientific research documents, whether they are published or not. The documents may come from teaching and research institutions in France or abroad, or from public or private research centers.
L'archive ouverte pluridisciplinaire HAL, est destinée au dépôt et à la diffusion de documents scientifiques de niveau recherche, publiés ou non, émanant des établissements d'enseignement et de recherche français ou étrangers, des laboratoires publics ou privés. 


\section{Impact of Robot Initiative on Human-Robot Collaboration}

\author{
Thibaut Munzer \\ Inria, Bordeaux Sud-Ouest, \\ France \\ thibaut.munzer@inria.fr
}

\author{
Yoan Mollard \\ Inria, Bordeaux Sud-Ouest, \\ France
}

\author{
Manuel Lopes \\ INESC-ID, Lisboa, Portugal \\ Instituto Superior Tecnico, \\ Lisboa, Portugal
}

\begin{abstract}
This paper presents a study on the impact of autonomy in the context of human-robot collaboration. We consider two conditions: i) a semi-autonomous robot that decides when to execute a supporting action, and ii) a support robot that has to be instructed of each action on a collaborative task. The semi-autonomous robot gradually learns how to support the human through experience. We found that users prefer the semi-autonomous robot and that the behavior was closer to their expectations despite them being more afraid of it. We also found that even if users noticed the robot was learning in one case, they wanted more autonomy in both conditions.
\end{abstract}

\section{CCS Concepts}

- Computer systems organization $\rightarrow$ Robotic autonomy; •Human-centered computing $\rightarrow$ User studies;

\section{Keywords}

Semi-autonomous; Collaborative robot; User study

\section{INTRODUCTION}

Today industrial robots are still restricted to highly repetitive tasks where they work separated from humans. However, due to new technological developments both in robot safety and artificial intelligence, robots might soon see a more widespread adoption. Robot safety has recently been greatly improved thanks to more compliant designs and better human acknowledgment. This is allowing robots to work in the same workspace as humans without the risk to harm them. In the same time, advances in machine learning make it possible to have robots that can learn new tasks from nonexpert human operators. It makes it economically possible to use robots for short-lived tasks.

Especially for collaborative tasks, there is still no definitive answer on how much autonomy is expected by the human coworker. This paper focuses on studying how humans react to a robot that can take initiative. We have created a

Permission to make digital or hard copies of part or all of this work for personal or classroom use is granted without fee provided that copies are not made or distributed for profit or commercial advantage and that copies bear this notice and the full citation on the first page. Copyrights for third-party components of this work must be honored. For all other uses, contact the owner/author(s).

HRI '17 Companion March 06-09, 2017, Vienna, Austria

(C) 2017 Copyright held by the owner/author(s).

ACM ISBN 978-1-4503-4885-0/17/03.

DOI: http://dx.doi.org/10.1145/3029798.3038373 semi-autonomous robotic system that responds to instruction but can take initiatives when it is confident about the action to realize based on previous experiences. We compare it to a robot without such capacity.

Systems that progressively learn a behavior from human expert have been previously proposed. In $[3,5]$, the user controls the robot. Once it stops the system will use past experiences to generalize to new situations. The user can take back the control at anytime to guide the robot when he judges the behavior is incorrect. Closer to what we propose, other works consider estimating the robot confidence to request guidance when the action to take is unsure [1].

The impact of robot initiative in human-robot collaboration has been previously studied. In [2], the authors studied the impact of autonomy for a scheduling and executing task and found that user preferred to let control to the robot for the scheduling part. The present work differs on the following aspects : i) we study increasing autonomy and ii) in a collaborative task where human and robot share the same workspace.

\section{USER STUDY}

We perform a user study to check the following hypothesis: i) users find the robot more useful when it is able to take some initiatives and ii) users are less comfortable and more afraid when working with a semi-autonomous robot.

The study presented the subjects sequentially with two conditions instructed and semi-autonomous. Half of the subjects started with the instructed condition while the other half started with the semi-autonomous condition. After each condition, the subjects answered to twelve questions, presented as Likert scales about their experience. Finally, they were asked which condition they preferred.

The task considered was to assemble a toolbox with the help of a Baxter robot. The user could instruct the robot to do support actions using an interface on a tablet. The robot is equipped with 4 actions. It can pick a piece, give a piece, hold a piece in place (to help the user screw) and reset arms in home position. The pick and give actions are realized with the left arm while the hold action is realized with the right one. The robot is controlled using the RAP framework [7] that allows concurrently running actions and uses relational representations for states and actions.

The semi-autonomous condition is composed of three assemblies of the toolbox. During the assemblies, the system was gathering information to learn a relational policy learner, in particular TBRIL [6], to learn a mapping from states to actions. It also uses Query by Bagging [4] to esti- 


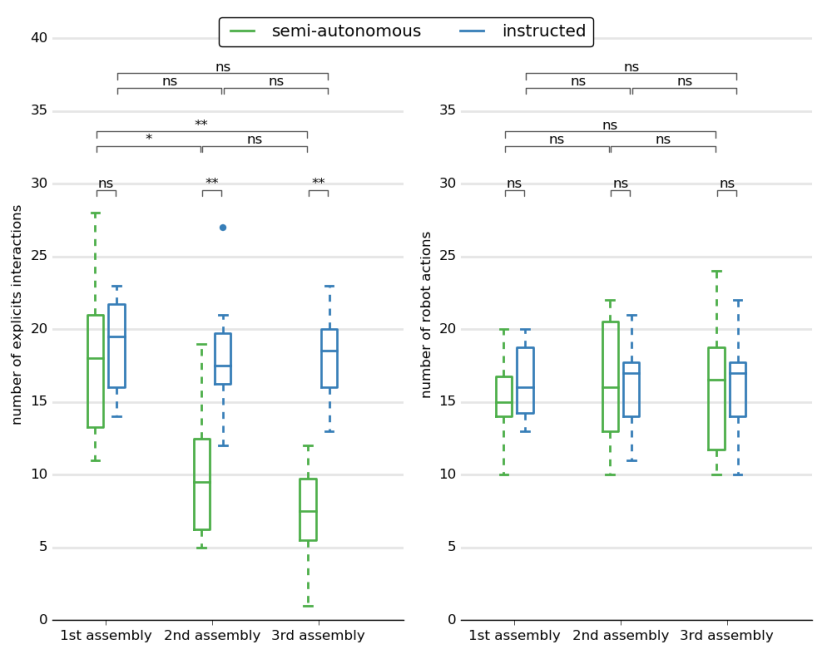

Figure 1: Number of interactions with the tablet (left) and number of action (right).

mate its confidence. During the second and third assemblies, each time it encountered a new state, it predicted the correct action and associated confidence. When the confidence was superior to a threshold, the robot started the action autonomously. Otherwise, it asked the user to confirm.

The instructed condition was also composed of three assemblies of the toolbox. However, no learning was involved. The user had to instruct all actions to the robot.

The study was conducted on 10 subjects ( 4 females) of age $30.1 \pm 10$. They self-reported an experience with robotics system at $3.1 \pm 1.6$ on a scale from 1 to 5 .

\section{RESULTS}

Figure 1 presents the number of interactions with the tablet and number of robot actions for both conditions. In the semi-autonomous condition the robot is able to learn the correct action, this can be seen as the number of interactions decreases with the number of assemblies while the number of robot actions stays constant. On the contrary, for the instructed condition both numbers stay constant. The number of interactions is sometimes higher than the number of actions because user tried to instruct actions before they were available (for example give, before pick finished).

Results of the questionnaires are shown in Figure 2. The first four questions are related to how helpful the robot is. In three of these four questions, the results are statistically significantly better for the semi-autonomous condition. They considered that the robot was a good coworker, that it made the task easier and that it was better to do the task with the robot. Also when asked which condition they preferred $90 \%$ of people choose the semi-autonomous condition.

The next four questions are related to acceptability. While users reported being more afraid of the robot during the semi-autonomous condition, they also reported that the behavior was more conform to their expectations. This means that these two criteria are not strongly correlated. We want to precise that due to precision errors the robot was sometimes failing its actions. We hypothesize that people would be less afraid of a robot making fewer mistakes.

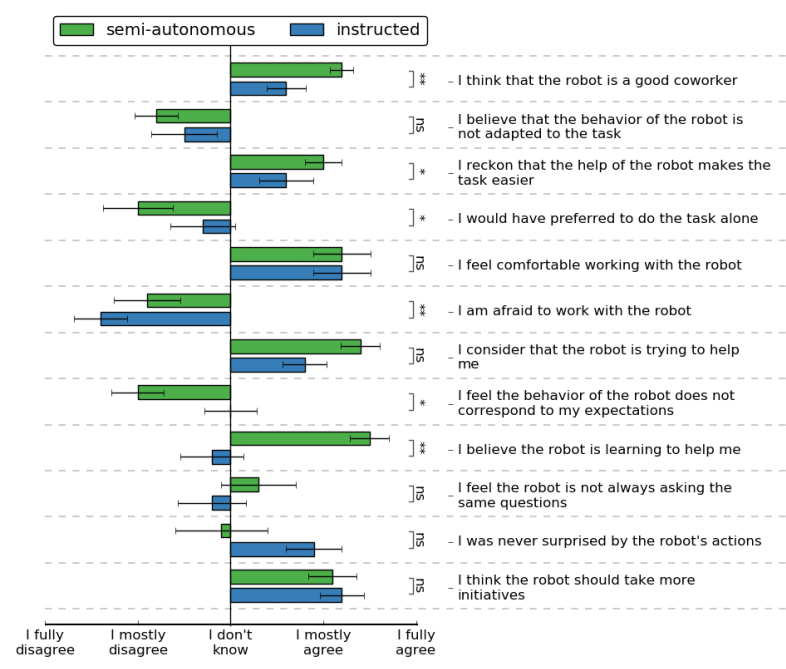

Figure 2: Answers to the questionnaire. Each subject experienced both conditions in random order.

The last four questions treat about autonomy and learning. Users clearly noted that the robot was learning in the semi-autonomous condition. The agreement to the question "I think the robot should take more initiative." is positive and similar for both conditions despite the robot taking no initiative in the instructed condition and starting half of its action by itself in the other conditions (see section 3 ). This means users are expecting more autonomy from the robot.

This paper presented a user experimentation to study the impact in terms of helpfulness and acceptability of a semi-autonomous robot for human-robot collaboration. The robot has shown to be seen as more helpful by the users. Users were also found to be more afraid of the semi-autonomous robot while its behavior was corresponding more closely to their expectations. As the users thought the semi-autonomous robot should take more initiative, future work includes comparing it to a fully autonomous (hard-coded) robot.

\section{REFERENCES}

[1] S. Chernova and M. Veloso. Interactive policy learning through confidence-based autonomy. Journal of Artificial Intelligence Research, 34(1), 2009.

[2] M. C. Gombolay, R. A. Gutierrez, S. G. Clarke, G. F. Sturla, and J. A. Shah. Decision-making authority, team efficiency and human worker satisfaction in mixed human-robot teams. Autonomous Robots, 39(3), 2015.

[3] D. H. Grollman and O. C. Jenkins. Dogged learning for robots. In ICRA, 2007.

[4] N. A. H. Mamitsuka. Query learning strategies using boosting and bagging. In ICML, 1998.

[5] M. Mason and M. Lopes. Robot self-initiative and personalization by learning through repeated interactions. In HRI, 2011.

[6] S. Natarajan, S. Joshi, P. Tadepalli, K. Kersting, and J. Shavlik. Imitation learning in relational domains: A functional-gradient boosting approach. In IJCAI, 2011.

[7] M. Toussaint, T. Munzer, Y. Mollard, L. Y. Wu, N. A. Vien, and M. Lopes. Relational activity processes for modeling concurrent cooperation. In ICRA, 2016. 